\title{
Ironie postmoderne et neutralisation des discours dans Parents et amis sont invités à y assister
}

\author{
Gabriel Proulx
}

Université McGill

Le roman d'Hervé Bouchard Parents et amis sont invités à y assister (2014 [2006]) se présente comme un récit complexe où il est difficile de se poser sur un passage et d'en tirer du sens, chaque phrase semblant recéler des contradictions, des obstacles à la lecture ou des renversements tantôt clownesques tantôt tragiques qui, bien qu'ils s'ancrent dans une tradition qui va de François Rabelais à Valère Novarina en passant par Samuel Beckett et Eugène Ionesco, sont en somme fort déstabilisants. Nous pouvons ainsi affirmer avec Stéphane Inkel que, dans ce livre atypique, « la narration est construite de telle façon qu'un 
indécidable affecte en permanence [les] monologues successifs, ce qui a pour effet de recouvrir d'un voile d'incertitude le moindre geste que le [...] roman nous propose » (2008, p. 34). Cette indécision des propositions langagières, discursives et littéraires, qui cause une incertitude chez le lecteur et un certain hermétisme textuel, rapproche bien entendu le texte de Bouchard des préoccupations postmodernes qui se lisent en filigrane dans une multitude d'œuvres contemporaines: fragilité des discours figés, fractionnement de l'identité auparavant monolithique des personnages, relativité des réalités spatio-temporelles, décloisonnement des langues, défiguration de la Belle langue, etc. Cette pratique, où toute réalité que l'on croyait stable semble se défaire de ses formes sclérosées pour mettre au jour la complexité du réel comme irréconciliable avec l'idée d’une langue fixée dans les discours fédérateurs de la société d'aujourd'hui, se présente donc comme ce que l'on pourrait nommer, avec Alan Wilde, une ironie postmoderne, c'est-à-dire une ironie « suspensive: an indecision about the meanings or relations of things [...] matched by a willingness to live with uncertainty, to tolerate and, in some cases, to welcome a world seen as random and multiple, even, at times, absurd ${ }^{1} »($ p. 44). Cette forme d'ironie recouvre entièrement l'œuvre de Bouchard, ce qui explique la difficulté qu'a le lecteur à résoudre tous les problèmes auxquels elle nous confronte, laissant ainsi flotter certaines questions dans une sorte de vide qui correspond à celui laissé par la neutralisation des grands discours qui régissaient auparavant la vision que l'on avait des événements et des réalités du monde. En effet, Bouchard défait l'une après l'autre chacune de nos certitudes, exposant par le fait même l'impossibilité de toute compréhension unidimensionnelle de la société, de ses tares comme de ses beautés. En donnant à son roman le sous-titre trompeur de « drame en quatre tableaux avec six récits au

\footnotetext{
1 « suspensive : une indécision face aux significations et aux relations qui unissent les choses, indécision couplée à un désir de vivre dans l'incertitude, de tolérer et, dans certains cas, d'accueillir un monde en apparence aléatoire et multiple, voire parfois absurde. » (Je traduis).
} 
centre » qui, en soi, ébranle les typologies génériques traditionnelles, en mettant en scène les discours toujours déjà déconstruits que sont les discours religieux et familial dont notre génération a hérité comme de déchets post-beckettiens impuissants et épuisés, et en triturant la langue normalisée afin de démontrer ses limites et les possibles qu'offre cette nouvelle langue instable, poétique et foisonnante, l'écrivain critique l'idée même de discours en y opposant non pas un autre discours prétendument supérieur, mais bien une hésitation totale. Selon Pierre Schoentjes,

[1]'ironiste serait [...] celui qui cherche un mode de communication plus authentique que celui de l'expression langagière normale. S'il feint ou dissimule et établit des rapports apparemment saugrenus, c'est parce qu'il cherche à établir, à travers la langue, un rapport neuf, tant avec la réalité qu'avec les autres; un rapport original dans lequel il aurait une part. Il lui faut pour cela rompre avec l'héritage du passé, rompre avec les autorités qui, avant lui, ont établi les rapports désormais canoniques et dont il se considère prisonnier. (1993, p. 248)

Dans le cas de Parents et amis, l'ironie postmoderne atteint se rapport à la réalité par le biais d'une fragilisation des discours clos qui a un effet double: la suspension du sens et l'indécidabilité discursive d'une part, et de l'autre, une multiplication des potentialités du texte et de la langue ainsi décloisonnés. Comme l'indique René Audet dans un article sur l'ironie de la forme, « la réutilisation décontextualisée de cadres discursifs [propres à un genre prédéterminé] produi[t] un effet de décalage sensible » (p. 17) une fois appliquée à un autre genre littéraire, décalage qui génère différentes lectures possibles de l'œuvre. Notre objectif sera par conséquent de déplier les dispositifs ironiques proposés par Bouchard et leurs effets dynamisants afin d'exposer leur caractère à la fois désorientant et fécond.

\section{Roman, théâtre et poésie : vers une hybridation générique ludique}

La première instance ironique à laquelle le lecteur est confronté est, comme nous l'avons observé, d’ordre générique. Cette ironie, que René Audet nomme ironie de la forme, 
correspond au fait de « [p]arler à travers les usages d'un autre genre » (p. 17) dans une œuvre appartenant explicitement à un genre connu du lecteur. Ici, Parents et amis, qui est en réalité un roman et a été étudié comme tel par la critique, recourt aux codes du genre dramatique, par exemple en se développant principalement par le biais de dialogues, en utilisant les didascalies et en nous proposant des décors qui nous rappellent ceux de la scène de théâtre. Il se présente comme un drame, mais cette hybridation du genre a pour seul objectif de déstabiliser le lecteur et de remettre en question la pertinence de telles étiquettes génériques, puisque les éléments théâtraux mobilisés sont tous déformés et rendus inopérants. L'idée même d'un drame au centre duquel l'auteur aurait inséré artificiellement des récits semble d'ailleurs d'ores et déjà suggérer que Bouchard déjoue les attentes du public en ce qui concerne la forme, étant donné que les problèmes de représentativité dramatique que montre cet assemblage des formes théâtrale (le drame) et narrative (les récits) sont nombreux. Selon Ginette Michaud, "[l]a question du genre est [...] l'un des enjeux importants du postmodernisme » (p. 71) et le traitement qu'en fait Bouchard est foncièrement expérimental, ce qui rapproche déjà ses préoccupations de l’esthétique postmoderne.

Dès le début de la lecture, les mécanismes dramatiques déraillent, ce qui a pour effet d'accentuer le décalage entre le sous-titre choisi par Bouchard et la nature réelle de son livre. Entre autres, les didascalies ne sont plus descriptives mais plutôt narrativisées, ce qui contrecarre absolument leur rôle, qui est à l'origine de donner, dans un langage extrêmement clair et épuré, des indications scéniques au metteur en scène et des instructions précises aux acteurs. Par exemple, on nous dit dès les première lignes du roman, que « [l]'un fait la liste des dans l'ordre où ils sont sans qu'on puisse le voir » (Bouchard, 2013, p. 11) : un personnage dont le nom n'est pas précisé, ce qui complique déjà le travail de mise en scène - ferait donc l'inventaire d'éléments dont la nature n'est jamais explicitée et ce même personnage sans nom 
est invisible. Dans une pièce de théâtre réellement destinée à être jouée, le nom du personnage et celui des objets ou des personnes dont il fait la liste seraient bien entendu donnés mais, surtout, les activités d'un personnage que l'on ne voit pas ne seraient pas mentionnées, puisque ledit personnage serait dans les coulisses et que ses activités ne devraient être rapportées que si elles avaient une incidence quelconque dans le déroulement de l'intrigue. Comme l'indique Laurance Ouellet Tremblay, cette pièce narrative idiosyncrasique «n'attend pas la venue d'acteurs réels pour révéler et assurer sa cohérence » (p.45). Bien entendu, ce type de détournement générique n'est pas sans rappeler les influences de Bouchard citées au début de cet article (Beckett et Novarina, en particulier), mais il exacerbe les mécanismes empruntés à ces auteurs de telle façon que l'aspect théâtral de l'œuvre en devient presque caricatural.

Bouchard lui-même a d'ailleurs décrit son livre comme une «pièce injouable, un roman théâtral plutôt» (2007), ce qui démontre la conscience qu'a l'auteur de la cassure irrémédiable qui existe entre l'étiquette qu'il appose à son texte et sa nature concrète. Tout au long des didascalies, on nous dit entre autres que « [l]es acteurs vieilliront » (Bouchard, 2014, p.11), mais on ne suggère aucun procédé technique ou scénique pour montrer leur vieillissement - que ce soit du maquillage, des effets d'éclairage ou le remplacement des acteurs par d'autres acteurs plus âgés durant la pièce. Cela sous-entend qu'ils devront vieillir naturellement, opposant donc au temps fixe et restreint de la pièce de théâtre la durée - par définition beaucoup plus grande - du roman et de la vie réelle. On nous explique aussi que «[1]es orphelins de père numéro un, numéro deux, numéro trois, numéro quatre et numéro cinq peuvent être joués par un seul et même » acteur (Bouchard, 2014, p. 12). Cependant, celui-ci devra être capable de les jouer tous en même temps, puisqu'ils parlent parfois en chœur, ce qui renforce l'impossibilité de représenter cette pièce, exhibe l'antiréalisme 
bouchardien et expose la fragilité de l'identité des orphelins en question. Dans le cas de l'épisodique Laurent Sauvé, il serait préférable, selon l'auteur, d'« engage[r] le fils d'un dieu » (p. 12), mais seulement si le théâtre en a les moyens financiers. La langue coupée du prêtre est censée être un personnage à part entière, ce qui soulève des questions d'ordre technique, puisque cette langue doit être détachée du corps auquel elle appartient et s'exprimer seule; «[l]es figurants du défilé initial sont une quarantaine environ [et] peuvent aller sans tête» (p. 12) de par la nature de leur rôle, et ainsi de suite².

Nous pouvons donc affirmer que le théâtre travesti de Bouchard est "un théâtre imaginaire parce qu'il n'existe et ne saurait exister que par le récit » (Camus, 2012, p. 140), ce qu'explicite d'ailleurs le romancier en insistant sur l'aspect artificiel de son drame lorsqu'il écrit : «La scène entière est crayonnée. La musique est crayonnée [...]. Les décors sont crayonnés» (Bouchard, 2013, p.12). Naissant par conséquent de l'écriture et y étant enfermée de façon définitive, la pièce-roman fait du surplace et n'arrive à s'extirper ni de ses allures de théâtre ni de sa nature foncièrement narrative, générant du même coup une forme hybride qui à la fois déçoit par le non-respect des promesses qui découlent de son titre et surprend par la richesse des propositions rendues possibles par son appartenance oblique à différents genres littéraires. Personnages, dialogues, décors, tous les repères traditionnels qui nous permettent de nous situer dans le genre dramatique semblent être réduits à un état essentiellement linguistique guidé par le simple crayon de l'auteur et non plus par l'intrigue ou l'action théâtrale et, «[d]ans ce théâtre par écrit, même les didascalies sont emportées dans le flot du langage » (Canty, 2007, p. 70).

\footnotetext{
2 Dans un court article sur les œuvres plus récentes de Bouchard, dont Le faux pas de l'actrice dans sa traîne, LouisDaniel Godin définit ces incongruités comme des « défis scénaristiques » et montre que ces derniers sont devenus une sorte d'obsession chez l'auteur saguenéen (p. 45).
} 
Les récits du centre n'échappent pas non plus à cette confusion générique, puisque certains d'entre eux prennent en réalité la forme de lettres ou de dialogues. Effectivement, le deuxième récit est explicitement présenté comme « une lettre [à ses fils] où elle [la veuve Manchée] parle des morts et de son dégoût des fleurs » (Bouchard, 2014, p. 95) et respecte parfaitement les codes du genre épistolaire, dont l'adresse initiale (« Chiens» [p. 95]) suivie de la virgule et le recours à la deuxième personne afin d'ancrer le texte dans un dialogue entre destinateur et destinataires. Le troisième récit, lui aussi épistolaire, se présente cette fois-ci comme « une lettre qu'ils [les orphelins de père] font à la mère Manchée » (Bouchard, 2014, p. 107) et, encore une fois, les codes du genre sont respectés, mais cette partie n'est elle non plus manifestement pas un récit à proprement parler. Le quatrième récit s'ouvre sur une sorte de prière prononcée par deux des fils qui, épuisés de vivre avec leurs tantes, expriment leur désir de quitter le nid familial, prière où les «missionnaires saisonniers », « les crucifiés », les « cieux» et la « $[\mathrm{m}]$ arde » (Bouchard, 2014, p. 119-120) se mêlent en une sorte de chant apocalyptique et où les frères semblent se poser tour à tour comme des damnés et des martyrs; s'ensuit un échange éclaté entre des personnages dont les noms ne renvoient à aucun des personnages croisés jusqu'à maintenant. Finalement, le sixième récit est une liste des pères imaginaires dont se réclame l'orphelin de père numéro six après son suicide, liste poétique qui semble plutôt se rapprocher de l'élégie que du récit, ce genre lyrique ancien étant bien entendu rendu de manière tout aussi ironique que le théâtre déthéâtralisé du drame que nous donne à lire l'écrivain.

Comme le dit Pierre Schoentjes, «il arrivait à Picasso de jouer sur le rapport [ironisant] entre le titre d'une toile et son sujet » (2001, p. 224) et un problème semblable de distanciation ou de rupture entre le signifiant et le signifié est établi par Bouchard dès la page couverture de Parents et amis, puisque le drame entrecoupé de récits qu'il annonce dans le 
sous-titre de son livre est très vite désamorcé par le biais du renversement des codes dramatiques et romanesques conventionnels. Cette trahison ludique du lecteur permet de «dynamiser la production du sens, [d']en empêcher la fixation» (Audet, 2013, p. 32) en forçant le lecteur à se questionner sur la nature des différents genres littéraires, tout autant que sur les voies qu'ouvre le métissage des dits genres. Les typologies génériques se posant comme des vérités rigides qui accueillent difficilement les genres hybrides qui se dressent contre les formes convenues, l'ironie de la forme permet de s'y attaquer par le biais d'une déconstruction des conventions et l'inscription volontairement problématique d'une œuvre complexe dans un genre précis qui ne saurait accommoder toutes ses particularités.

\section{Le Christ, le père et la mère : neutralisation des discours fédérateurs}

Tout comme la réflexion sur les genres littéraires et les discours réducteurs qui en découlent, les discours sociaux n'échappent pas à l'ironie bouchardienne. Cependant, les discours religieux et familial ne sont pas réellement déconstruits par l'auteur; ils subsistent sur scène, dès le début du texte, comme des réminiscences déjà déconstruites d'un monde révolu et n'offrent aucune réponse aux questions des personnages par rapport à leur avenir ou aux choix qu'ils doivent faire, si ce n'est qu'ils mettent au jour la faillibilité et l'imperfection de l'homme de poussière qui, inévitablement, devra vivre dans la boue et y retourner.

Le discours ecclésiastique est montré, dès le premier tableau, dans toute son impuissance par le biais du personnage du prêtre. En effet, ce dernier nous est présenté comme un être mutilé dont la langue coupée ne cesse de gigoter et de répéter les mêmes paroles, qui n'ont aucun impact - négatif ou positif - sur les autres personnages. Alors que cette langue littéralement désarticulée tente de réunir les proches du défunt autour de la parole religieuse, aucun d'entre eux ne répond à son « Dijons une çapelet » (Bouchard, 2014, 
p. 18, 20, 24 et 33). Cette parole fragile et vide, c'est donc celle d'une religion qui n'est plus perçue comme une force rassembleuse et réconfortante qui permettrait de réunir dans une même communion tous les membres de la famille à la suite d'un événement marquant - en l'occurrence, la mort du père -, mais plutôt comme une répétition mécanique et impotente d'un refrain qui a perdu tout son sens et qui, par le fait même, est prononcé en zozotant silencieusement. Plus loin dans le récit, c'est d'ailleurs la phrase «Manhez-en tous» (Bouchard, 2014, p. 171), tout aussi courte et insignifiante, qui remplacera la première, ce qui n'aura pas plus d'influence sur les autres personnages et leurs actions. À la force du discours religieux et à l'importance des curés dans la société québécoise d'antan s'est donc substitué un déchet discursif qui ne fait que flotter et s'écouter parler, totalement inconscient de sa déchéance et pourtant sur le point de disparaître, ses bégaiements se rapprochant de plus en plus d'un simple silence.

Quant à lui, l'alpiniste reconnu pour être prêtre se présente comme une alternative quelque peu déjantée mais aussi plus connectée au réel que ce premier prêtre charcuté. Effectivement, dans son discours, il semble avoir pris conscience des changements qui ont eu lieu dans la société à laquelle il s'adresse. Cependant, qui dit changement dit parfois trahison, puisque ces idées s'opposent souvent à celles que prône l'Église. Par exemple, au cours du troisième tableau, il explique que Jésus était autant le défenseur des femmes adultères, que nous admirons pour sa vertu, sa générosité et son empathie (Bouchard, 2014, p. 192), que le larron qui fit son entrée à Jérusalem « sur un âne volé » (p. 191), qui se mit en colère contre un figuier quand ce dernier ne lui donna pas de fruit (p. 193) et qui exigea de se faire oindre d'albâtre malgré le fait que cette substance dispendieuse aurait permis, si on l'avait vendue, de soulager les pauvres (p. 197-198). Il nomme d'ailleurs Jésus «notre Seigneur le grand J» (p. 189), «grand J» ou encore simplement «J» (p. 191-192) ce qui, déjà, rompt avec la 
glorification traditionnelle de la figure christique et rapproche les préoccupations du Christ de celles des petites gens dont il est question dans Parents et amis. Ce renversement de la figure du Christ de l'axe transcendantal, c'est-à-dire de son statut de fils direct de Dieu et d'exemple de piété et de charité, à l'axe de l'immanence, soit celui des simples mortels, recontextualise le discours religieux en le nuançant. L'alpiniste « valorise [donc] le doute, assume la contingence du sens sans jamais accepter l'imposition d'une Vérité "fondamentale" ou "essentielle" » (Adriaensen, 2004, p.98), ce qui neutralise le grand discours ecclésiastique, ou plutôt le minorise en y faisant entrer une part de doute et de sincérité contraire à la notion obsolète d'idolâtrie aveugle.

L'alpiniste conclut la partie de son allocution portant sur Jésus avec une déclaration beaucoup plus explicite de cette idée d'un Christ imparfait et éloigné de la construction unidimensionnelle qu'en donne l'Église :

Chaque fois, mais c'est toujours la même fois, chaque version de l'histoire montre que notre J se met au-dessus des pauvres d'une manière qui choque notre idée d'un dont l'humilité est gage de grandeur. Tombons sur le cul, oui. Par cette conduite, il semble se ranger dans la lignée des prêcheurs d'aujourd'hui, qui commandent le dépouillement et l'humilité cependant qu'ils vont en limousine grâce aux dons qu'on leur fait. (Bouchard, 2014, p. 197-198)

Ici, Jésus est directement comparé aux preachers, ce qui ramène la religion chrétienne à son caractère originellement sectaire et déforme l'image immaculée du fils de Dieu en le rendant plus humain que bien d'autres hommes simples qui «répandent la joie et la vie pour rien» (p. 198) dans un anonymat et un refus du culte de leur personne par autrui. Jésus entrant à Jérusalem sous les applaudissements avec son âne volé - l'équivalent de l'époque selon le texte d'une limousine payée à même les dons de ses fidèles - serait donc un coup de marketing comparable aux émissions de télévision américaines mettant en vedette des révérends exubérants aux opinions tranchées. Cette observation pousse finalement l'alpiniste à sommer ceux qui sont réunis autour de lui de «tomber sur le cul », sorte d'appel à une 
communion corrompue qui remplacerait les mains jointes de la prière et l'agenouillement catholique.

Ces renversements carnavalesques sont très nombreux dans l'œuvre de Bouchard. Par exemple, les noms de paroisse inventés de toutes pièces comme «Saint-Flatulon-du-DerrièreCoulant» (p.185), dans lequel les flatulences et le bas corporel sont littéralement mis en relation avec la sainteté, ou encore «Hosties-de-Saints-Tombant-de-Haut», où la chute des saints du haut de leur tour d'ivoire est littéralisée, montrent à quel point l'écrivain souhaite rompre avec la religion monolithique qu'est le christianisme en ébranlant et en travestissant les croyances rigidifiées et sclérosées qui la régissent. Jésus, auquel l'alpiniste s'attaque sans relâche, comme nous venons de le voir, est d'ailleurs réduit à ce même état de bassesse morale, puisque l'alpiniste explique « que celui que nous mangeons symboliquement depuis deux mille ans était un hostie déjà de son vivant» (Bouchard, 2014, p. 198). Cela a un effet double : premièrement, le rapprochement du juron québécois « hostie » et de l’hostie comme représentation du corps du Christ et, ensuite, d'introduire cette hostie désacralisée dans le système digestif, causant donc une permutation de la symbolique eucharistique, selon laquelle le corps de Jésus pénétrerait celui des fidèles afin que les valeurs de bonté et de charité du Christ les pénètrent tout autant, vers une conception totalement corporelle de l'ingestion d’une hostie dépouillée de sa charge métaphorique qui passerait par la bouche pour finir par être digérée et expulsée du corps comme tout autre aliment. L'alpiniste dit aussi du suicidé qu'il « partit, pua, revint » (p. 199), énumération dans laquelle François Ouellet voit une autre inversion du haut et du bas : «Cette formule en trois temps oppose une vision carnavalesque de l'humanité à celle, héroïque, de Jules César: “veni, vidi, vici” (je suis venu, j’ai vu, j’ai vaincu)» (p. 209), preuve que la force ironique du carnavalesque contamine toute forme de 
discours qui cherche à créer une scission irrémédiable entre le divin ou le grandiose, d'une part, et de l'autre, l'humain ou le petit.

Autre pilier traditionnel de la société chrétienne - et, dans le cas de Parents et amis, québécoise -, la famille et les valeurs de cohésion qui y sont normalement associées sont aussi mises à mal par Bouchard. Or, tout comme le livre s'ouvre sur la langue déjà coupée et le discours désamorcé du prêtre, la mort du père se présente comme un événement antérieur au temps de l'action théâtrale, puisque nous assistons dès le premier tableau à ses funérailles. La figure du père, que l'orphelin de père numéro six tentera de retrouver parmi tous les hommes qu'il a croisés durant sa vie dans le dernier des six récits du centre, est donc absente et inopérante, voire spectrale, puisqu'elle hante le texte mais comme une réalité disparue à tout jamais et non une réalité tangible et accessible aux orphelins et à la veuve. «[L]a mort que trouve Hamlet à la fin de la pièce [de William Shakespeare], elle est d'entrée de jeu le lot des fils de Parents et amis » (Ouellet, 2010, p. 206), ce qui signifie que cette tragédie initiale de la perte du père ne mènera pas les orphelins vers une réelle quête de sens; le voyage initiatique est plutôt remplacé par une mort symbolique immédiate des orphelins, ces derniers étant condamnés à leur état d'animalité et ne pouvant jamais plus franchir la ligne qui sépare l'enfance farouche de l'âge adulte.

D’ailleurs, le voyage qu'entreprennent deux d'entre eux vers l'Ontario afin de se trouver un emploi et d'échapper à leurs tantes les amène à faire face à leur développement interrompu par le départ du père - encore une fois une figure empreinte de connotations religieuses fortes. En effet, l'un des deux orphelins voyageurs écrit qu'on leur a expliqué, alors qu'ils se cherchaient un emploi, que « chauffeur de tracteur c'est moins dur et c'est plus difficile et t'es moins un homme à la fin » (Bouchard, 2014, p. 131), ce qui explicite l'objectif de 
cette échappée hors du foyer familial : devenir un homme ${ }^{3}$, malgré l'absence du père qui était censé mener, par l'exemple, à ce processus social attendu qu'est le passage de l'enfance à l'âge adulte. Néanmoins, leur quête n'aboutit à rien, puisqu'ils écrivent plus tard : «assis nos pieds ne touchent pas le sol. C'est dire combien ne sommes alors pas des hommes » (Bouchard, 2014, p. 133). Leur tentative d'entrer dans un bar se soldera aussi par un échec, car le portier leur dira de vieillir s'ils veulent boire, mais il leur refusera toujours l'entrée - cette fois violemment - quand ils reviendront plus tard: «Nous vieillissons, c'est long, puis nous entrons. Long Tarien en christ, sachant pas boire, pète son dentier en me boxant. Partons courant» (Bouchard, 2014, p.129-130). Les orphelins sont non seulement incapables de devenir des hommes à part entière de par l'absence d'un guide paternel pour les mener à cet état de maturité, mais aussi d'accéder au langage de l'adulte, puisqu'ils prennent au pied de la lettre la demande du portier et sortent du bar pour un moment afin de vieillir. Comme l'enfant qui prend la montagne entre ses doigts au loin en fermant un œil et croit par le fait même cette montagne toute petite, les orphelins n’ont aucune maîtrise des échelles de grandeur et des systèmes qui permettent d'organiser notre compréhension du monde, dont fait bien entendu partie le temps. Alors que le portier leur dit évidemment de revenir lorsqu'ils auront atteint l'âge légal pour consommer des boissons alcoolisées, ils comprennent plutôt qu'il leur suffit d'aller vieillir de quelques minutes ou de quelques heures avant de revenir, leur rapport à la langue étant par conséquent celui de l'in-fans, c'est-à-dire de celui dont la langue n'a pas encore été réglée et assagie par la société, et il semble que la mort de leur père les prive de cette accession à une langue commune qui leur permette de s'ancrer efficacement et

\footnotetext{
3 La vision de la masculinité dont il est question ici reste très traditionnelle : un homme doit à la fois être un pourvoyeur (" chauffeur de tracteur, ça rapporte moins, cinq dollars de moins par jour ») et travailler fort physiquement («Ce n'est pas qu'il soit difficile de conduire un tracteur »). Être chauffeur de tracteur est donc à la fois plus difficile parce qu'il faut parler anglais et être prudent afin de ne pas causer d'accident, et plus facile parce que cela ne requiert aucun talent manuel particulier; le métier d'«accrocheur de bâtons » est par conséquent préférable, car cela est plus payant et plus exigeant physiquement, ce qui représente les deux piliers de la masculinité telle qu'elle apparaît aux orphelins.
} 
adéquatement dans la société qui les entoure. Comme le suggère David Beaudin-Gagné à propos du père Beaumont, « son absence entraîne la régression de[s] [...] orphelin[s] vers une étape antérieure de l'enfance, limitée aux besoins les plus primaires ("j'ai faim, je vais vomir, je vais mouiller mon pantalon") » (p. 64), régression physique et psychique à laquelle répond une certaine stagnation langagière.

La relation de la veuve Manchée avec ses fils est tout aussi problématique que celle qu'entretiennent les fils avec leur père disparu. Dans la lettre qu'elle leur adresse dans le deuxième récit du centre, par exemple, elle les apostrophe, comme nous l'avons vu précédemment, en les appelant «Chiens », et la quatrième de couverture du roman nous indique qu'elle « s'adresse [...] à ses fils les chiens à tête de veau » (Bouchard, 2014, quatrième de couverture), ce qui démontre la haine qu'elle a pour ces éternels enfants au caractère bestial et indomptable. Ce dégoût, elle l'avait aussi pour son premier enfant, qu'elle et son mari le père Beaumont « [se] sent[irent] contraints de [...] jeter en syndrome de mort né jeune afin d'échapper à ce mal si mal dit par le prêtre » sans langue (p. 98), sorte de prophétie d'un enfant qui «ferait ce qu'il veut de sa queue, c'est-à-dire nettoyer des culs », qui laisse planer l'idée d'une homosexualité - ou d'une déviance - annoncée du premier rejeton en quelque sorte tué dans l'œuf. Nous pouvons donc affirmer que, au-delà de la relation conflictuelle qu'elle entretient avec sa progéniture, la veuve Manchée a une relation difficile avec l'idée même de maternité. En effet, alors qu'elle raconte la mort de l'un de ses cousins à ses fils, elle dit s'être rendue «rue Pourrissons, rue Nourrisson, [...] rue Morisson» (Bouchard, 2014, p. 100), suite où l'on entend tour à tour résonner la pourriture, l'enfant sans défense de la

\footnotetext{
${ }^{4}$ La mort du père - tout comme la famille dysfonctionnelle de façon plus large - est une thématique littéraire que l'on retrouve dans des œuvres aussi éloignées que le Hamlet de Shakespeare et La petite fille qui aimait trop les allumettes de Gaétan Soucy. Cependant, la mère anti-maternelle est une figure encore assez taboue et peut être considérée comme contre-nature (pensons à L'obéissance de Suzanne Jacob). La Veuve manchée n'est donc pas la première mère du genre dans la littérature québécoise, mais ses paroles et son attitude n'en restent pas moins choquantes.
} 
période néonatale et la mort. Or cette peur de la reproduction provient d'une peur plus profonde de la mort ou, plus précisément, de la non pérennité de la vie; c'est effectivement le fait de savoir que, dans un univers sans Dieu où la langue du christianisme pend mollement dans le vide et que les repères qui nous avaient jusqu'à maintenant permis de nous situer dans le monde n'ont plus aucune autorité, «être sauvé, c'est apprendre sa mort, que nous sommes mortels, que nous sommes tous sans père » (Ouellet, 2010, p. 213) au sens religieux comme familial, et cette connaissance de l'insignifiance d'une vie menant imparablement à une mort toute physique et profondément charnelle rend d'autant plus difficile de voir la naissance d'un nouvel être humain comme un événement à célébrer. "Ma haine, c'est un jeu, c'est une offrande que je vous fais en vous disant de manger de la marde. Je suis au centre et j'y suis sans douleur [...]. Vous êtes les fruits de mes entrailles » (Bouchard, 2014, p. 210), nous dit la veuve Manchée dans le troisième tableau, ce qui montre que sa méchanceté n'est qu'une échappatoire à la tristesse et à la douleur profondes qui naîtraient inévitablement d'une acceptation totale de la médiocrité de la vie et de la mort. Selon Laurance Ouellet Tremblay, « la veuve ouvre [...] la bouche non pour prédire ce que le futur réserve aux siens, mais bien pour rappeler ses fils à leur propre histoire et à la nécessité de définir le rôle de leur vie » (p. 83), rapprochant donc le discours de la veuve de l'invective. Nous y voyons plutôt un exemple du complexe de la mère morte, c'est-à-dire que « [p]résente et vivante, dévorée par une dépression sévère (liée à un deuil [...]), cette mère est subitement trop triste pour s'intéresser de façon vivante à son enfant » (Estellon, 2013, en ligne), ce qui expliquerait la nature de ses échanges avec les orphelins. Barrière entre elle et la violence du monde et de son propre cercle social, l'inimitié de la veuve serait en réalité un mécanisme de défense. 


\section{Le langage malmené, ou quand la langue ne veut plus rien dire}

Bien que le traitement ironique - et par conséquent critique - d'enjeux sociaux et artistiques soit au cœur de l'œuvre de Bouchard, il est important de noter que cet ébranlement des (fausses) vérités sécurisantes qui donnent à l'être humain la chance de se construire une identité en apparence cohérente et immuable s'incarne aussi directement dans la langue bouchardienne. Le détournement poétique et coloré de la langue standard se présente comme une attaque à l'un des principaux bastions de notre civilisation, puisque la langue elle-même n'est souvent pas considérée comme une instance de contrôle artificielle. En effet, alors que le discours religieux a perdu de sa dorure et que les sociétés occidentales semblent se détourner de plus en plus de celui-ci, le langage est souvent vu comme un simple véhicule, mais Bouchard nous en donne une version détraquée qui le fait surgir dans ses soubassements sauvages et déformants.

L'auteur multiplie entre autres les effets et les inventions de langue : les jeux de mots («Papa mort, il y a deux ans, fut emporté par le cancer du récipient» [Bouchard, 2014, p. 211]), les pléonasmes steiniens (« Tous les morts qu'il connaît sont morts et il les connaît » [p. 36]), les échos phonétiques («Quel sommeil de corps [...] / Quel sommeil découdre / Quel décor soumettre / Quel sommeil découdre» [p. 205]) et les barbarismes («Sacrons pour légender l'hurlurlement de nous qui sommes orphelins » [p. 33, je souligne], l'Ontario qui devient l’« Onratorion » [p. 129]) défigent les sens et font entrer dans la langue normalisée un souffle nouveau, que l'on pourrait qualifier de souffle de folie ${ }^{5}$. Quant à elle, la versification (« Je sais pas / Mais je suis triste, ouais / Je voudrais aller là où je suis pas allée / Rester là et /

\footnotetext{
5 Cette langue correspond assez bien à celle que décrit Gilles Deleuze dans Critique et clinique, c'est-à-dire à « un devenir-autre de la langue, une minoration de cette langue majeure, un délire qui l'emporte » (p. 15). La langue poétique de Bouchard, à l'instar de celle d'auteurs assez proches tels que Réjean Ducharme, déborde sans arrêt et c'est dans ses débordements, ses excès et ses logorrhées, entre autres, que se lisent les conflits sociaux et familiaux qui habitent l'œuvre.
} 
Me couler dans ton corps où la nuit entre pas / Mais nous sommes séparés / Dans le soir frais de mai / Et je prie, ouais [...]» [p. 63] ou encore « Je n'ai trouvé la mort au bout de ma vie / Je n’me suis pas relevé tout seul / Je n'ai trouvé la mort au bout de ma vie / Ma vie, ma vie n'est pas ma vie [...]» [p. 200]) donne à certains passages des allures de chanson populaire avec ses marques d'oralité et son rythme, ce qui participe du mélange des genres et de la rencontre de différents niveaux discursifs. Pour leur part, les répétitions (le « Merci d'être venu » [p. 14] répété une quarantaine de fois au salon mortuaire, la fausse accumulation dans « il avait tout mangé, il avait tout mangé mangé, il avait tout mangé mangé mangé, [...] il avait tout mangé mangé mangé mangé mangé mangé mangé mangé mangé mangé mangé mangé mangé » [p. 178-179]) font tourner à vide les propositions et donnent l'impression que l'on avance du moins typographiquement, le texte s'allongeant par le biais de ces accumulations - alors que l'on ne fait que ressasser les mêmes mots sans fin. Dans l'accumulation du terme « mangé » dans la dernière citation, Laurance Ouellet Tremblay voit « un outil de dévoration du monde » (p. 93), ce qui signifie que ce procédé viserait une certaine totalisation du monde. Or, comme pour la formule « Rose is a rose is a rose » de Gertrude Stein, il est aussi possible d'y voir un épuisement du sens, voire un évidement de la langue à proprement parler. Ce type de répétition se présente par conséquent comme un mécanisme supplémentaire de l’ironie postmoderne bouchardienne; chaque énoncé de ce genre pouvant être lu de deux façons totalement opposées, le discours se situe constamment à la limite entre sens et non-sens, entre prolifération et annihilation, laissant par le fait même la parole « uncontrollable and deceitful $^{6} »$ (Hutcheon, 1986-1987, p. 192) se multiplier et se contredire en cours de route.

Cette défiguration de la langue a cependant des incidences plus importantes quant à la trame narrative et à la possibilité pour le lecteur de s'y situer. Entre autres, les repères spatio-

\footnotetext{
6 « incontrôlable et trompeuse ». (Je traduis).
} 
temporels auxquels le lecteur a normalement accès en régime narratif sont vidés, du moins partiellement, de leur signification7, ce qui fait flotter le récit dans un vortex détaché du monde. Il est ainsi impossible de savoir à quelle époque a lieu le récit que construit Bouchard ou de déterminer l'âge des personnages. Par exemple, Rogère, la sœur de la veuve Manchée, dit être «née en mil six cent quelque cent quelque dix et quelque six » (Bouchard, 2014, p. 68), année déconstruite qui n’a plus aucun sens. De plus, le temps ne semble pas se dérouler normalement, mais bien de façon aléatoire. Lorsque la mère Manchée parle de son petit dernier, elle dit : «Je l'entends prendre des centimètres, faut faire vite, il va parler, il sera trop tard » (Bouchard, 2014, p. 58). Ce passage démontre premièrement la peur qui habite la mère de voir son plus jeune parler, comme si le langage grossier et animal des cinq autres enfants de la famille représentait un état langagier inacceptable et honteux, mais il montre aussi que le temps tel que nous le connaissons, c'est-à-dire en tant que réalité mesurable et ordonnée, n'a plus cours; il a été remplacé par un temps douteux qui accélère et décélère librement selon l'importance de la place qu'occupe l'action en question dans la trame narrative, ce qui empêche le lecteur de se situer clairement dans l'espace-temps. De drôles de déductions naissent aussi de ce rapport aliénant au temps objectif. Dans sa lettre à ses fils, la veuve Manchée explique par exemple que, pour savoir quel âge elle avait lors de l'enterrement de ses cousins, elle doit connaître l'âge du cousin décédé : «j'avais huit ans, j'avais six ans, ça dépend de l'âge du mort» (Bouchard, 2014, p. 103), ce qui expose le rapport complexe et totalement relatif qu'elle entretient avec la temporalité. L'importance des chiffres dans l'univers de Bouchard est explicitée dès la première réplique du roman théâtral : «Sans les

\footnotetext{
7 Stéphane Inkel met en relation le travestissement de la religion et la mise à mal des repères temporels : «ce qui à première vue semble un accroissement du nihilisme s'avère au contraire une toute nouvelle manière, rigoureusement conséquente, de mettre en question le temps de l'histoire suite au dépérissement de l'autorité affectant la tradition (catholique)» (2009, p.128). Cette lecture nous semble particulièrement pertinente, puisqu'elle illustre bien comment l'ironie postmoderne permet de remettre en doute toute notion de certitude, le temps et la tradition devenant par le fait même malléables.
} 
nombres, on ne sait rien » (p. 13) et, en effet, la déconstruction des nombres significatifs, qui passe d'un côté par leur suppression et de l'autre par leur réduction à un état dégradé qui les rend irrécupérables, défige la signification du texte et donne l'impression d'un récit qui « est sans début ni fin » (Canty, 2007, p. 78).

« [L]'ironie propose une interrogation et s'efforce de mettre les vérités en question » (Schoentjes, 2001, p. 219), et cette remise en question de réalités que l'on croyait objectives et, par le fait même, inattaquables, se présente en définitive comme une force équilibrante qui ramène tous les discours à un même niveau sans en privilégier un en particulier, mais aussi déstabilisante, puisqu'elle donne l'impression que nous ne pouvons plus nous accrocher aux béquilles discursives qui gouvernaient auparavant notre façon de penser et qui façonnaient notre rapport au monde extérieur. Tout comme le concept de temps, et donc de temporalité, est bouleversé par l'écriture bouchardienne, le lieu où se déroule l'histoire de Parents et amis n'est jamais donné précisément. Alors que certains repères permettent de nous situer dans la région du Saguenay-Lac-Saint-Jean - les deux orphelins en fugue vers l'Ontario tentent en premier lieu de trouver du travail au Centre d'emploi de Jonquière (Bouchard, 2014, p. 115) -, nous sommes tenus dans une curieuse incertitude quant à la position géographique précise de la maison des Beaumont. La ville de Chicoutimi, autre indication géographique d'importance dans le récit, est tour à tour appelée «Chicoute» (Bouchard, 2014, p. 124), "Chicouti », «Chicon » et «Chicoutimou-Norne » (p. 125-126) et la malléabilité de son nom s'accompagne d'un doute sur le lieu réel auquel chacune de ses appellations se rapporte et sur la vraisemblance de l'histoire qui nous est contée. Le récit a-t-il vraiment lieu près de Chicoutimi, ou a-t-il lieu dans un lieu qui rappelle simplement cette ville, mais dont la cartographie serait reconfigurée par l'acte créatif et linguistique auquel s'adonne l'auteur? 
Est-ce vraiment la ville que nous connaissons, ou une sorte de double «crayonné » qui n'existe que dans, par et pour la fiction ${ }^{8}$ ?

Ces questions nous mènent au problème récurrent de la nomination dans le roman de Bouchard. Dès le début du texte, l'écrivain nous présente des orphelins qui n'ont pas de noms propres et qui, par conséquent, sont simplement nommés selon l'ordre dans lequel ils sont nés : leur identité est donc basée sur le fait qu'ils sont orphelins de père, ce qui est peu révélateur en ce qui a trait à leur caractère, et leur position respective dans l'arbre généalogique. De plus, leur mère est appelée, à différents moments du récit, «la veuve Manchée », « la mère Manchée », « Laïnée » et « Laïnalinée », ce qui rend difficile la fixation de son identité dans ce qu'elle a de plus fondamentale, soit son nom propre. Pour sa part, «l'épisodique Laurent Sauvé » devient simplement «Laurent Sauvé » lorsqu'il commence à occuper une plus grande place dans l'histoire, comme si l'adjectif décrivant son importance dans l'œuvre était d'une conséquence telle qu'il fallait l'apposer à son nom tant et aussi longtemps qu'il resterait littéralement épisodique. Les sœurs de la veuve Manchée ont quant à elles des noms propres, qui nous sont révélés sporadiquement durant le récit, mais l'auteur s'y réfère principalement en les appelant première sœur, deuxième sœur et ainsi de suite; leurs noms semblent donc n'être qu'accessoires, comme s'ils avaient été greffés artificiellement à ces personnages à la fois nommés et innommables, preuve supplémentaire de l'arbitraire du signe9.

\footnotetext{
${ }^{8}$ Il est difficile de ne pas faire le lien ici avec le texte de Stéphane Mallarmé intitulé « Crayonné au théâtre » (dont le titre se retrouve, comme nous l'avons vu, dans l'idée de la scène crayonnée suggérée par Bouchard au début de son roman), où il est question d' " un art, l'unique ou pur qu'énoncer signifie produire » (p. 157). Selon les deux auteurs, l'écriture ou la parole donneraient naissance à un monde réel, quoique n'existant que par le texte ou la voix : « L'auteur ou son pareil, ce qu'ils voulaient faire, ils l'ont fait » (Mallarmé, 1897, p. 153-154), et l'on pourrait même dire qu'ils l'ont fait advenir.

9 Pour une analyse des mécanismes et des effets propres à la liste de figurants qui clôt l'œuvre, voir Ouellet Tremblay, 2016, p. 52.
} 
Cette impuissance de la nomination à réellement faire sens pour les personnages et les lieux qui s'accumulent tout au long du roman rejoint l'allocution de l'alpiniste reconnu pour être prêtre, où ce dernier cite différents personnages bibliques nommés Lazare, qui semblent tous avoir eu la lèpre mais qui, malgré le nom et la maladie qu'ils ont en commun, ne sont pas la même personne. D’ailleurs, Lazare devient « Éléazar son nom en plus long » lorsque celui-ci est décrit comme « le célébré, l’aidé de Dieu », mais il devient au contraire « Elzéar son nom en plus proche » quand il est plutôt « l'affreux [...], le mort pas mort» (Bouchard, 2014, p. 189). Le nom bouchardien, élastique et mobile, s'allonge donc ici pour signifier la grandeur et la sainteté tout comme il se ratatine pour signifier la monstruosité et la maladie. Comme nous le dit l'orphelin de père numéro deux, «[l]es noms sont des tares auxquelles il faut s'habituer sous peine de perdre sa place dans le défilé » (p.177), c'est-à-dire que le fait d'avoir un nom est un couteau à double tranchant : d'un côté, avoir un nom propre avec lequel s'identifier fige la personne dans un moule - d'où l'appellation de «tare » - et, de l'autre, la liberté de n'avoir aucun nom est paralysante, car elle empêche de faire partie des rituels de la société à part entière et elle tend à freiner l'élan naturel de définition de soi, l'acte de se nommer étant à la base de l'édification d'une identité parfaitement formée. C'est ce qui fait des orphelins de père des coquilles vides où l'on ne lit que la détresse de l'adolescence informe et l'incapacité à se forger une identité pleine et mature, de par l'absence du père et du nom propre fédérateur. Les personnages de Bouchard sont ainsi «[e]ntièrement présents à la parole, ils sont sans intériorité autre que celle de la langue qui leur donne lieu »(Canty, 2007, p. 67), ce qui fait d'eux de simples pantins crayonnés par l'écrivain (Kemeid, 2007, p. 85) et, conséquemment, incapables de se détacher de la représentation mutilée et laconique qu'en donne ce dernier. Ce sont des «persons», comme le dit Bouchard, c'est-à-dire des personnes littéralement 
tronquées à qui il manque quelques lettres, un nom ou certaines parties du corps pour être complètes.

L'œuvre de Bouchard, qui semble constamment sur le point de se délier en raison de son indécision et des flottements qui rendent impossible la formation de toute idée arrêtée et de toute action totalement accomplie, est maintenue ensemble par une ironie postmoderne, que l'on pourrait aussi nommer une ironie baroque, selon la définition qu'en donne Barthes : «Face à la pauvre ironie voltairienne, produit narcissique d'une langue trop confiante en ellemême, on peut imaginer une autre ironie, que, faute de mieux, l'on appellera baroque [...], parce qu'elle épanouit le langage au lieu de le rétrécir»(p.76-77). Par conséquent, la contestation que fait l'auteur des notions de genre littéraire, de la toute-puissance des discours toujours déjà annihilés que sont la parole religieuse et les valeurs familiales traditionnelles et de la fixité de la langue comme moyen de communication transparent et inoffensif se présente comme une opposition à l'idée même de discours figé et de vérité. Selon Dominique Viart, « le discours lui-même, quel qu'il soit, ne peut plus être reçu comme vrai, [...] tout récit, légitimant ou non, a perdu de sa crédibilité en raison même d'une perte de crédibilité du langage»(p.120), et la double représentation de la perte de légitimité des discours fédérateurs et de la faillite du langage est au cœur du projet bouchardien. Au lieu d'opposer - selon la méthode voltairienne - un ensemble clos de valeurs nouvelles à un ensemble tout aussi restreint et hermétique de valeurs qu'il considérerait désuètes ou injustifiées, l'ironiste postmoderne s'affaire plutôt à mettre au jour la dangerosité de générer et d'entretenir les discours contraignants qui tentent de répondre sans nuance aux problèmes et aux réalités pourtant mouvants et pluriels auxquels l'homme se heurte au quotidien. Aux typologies génériques monolithiques et archaïques se substitue une libération de la forme et un détournement ludique des attentes du lecteur; aux discours ecclésiastique et familial se 
substitue une remise en question de ce qui guide maintenant l'humain dans sa quête de sens;

et à la Belle langue se substitue le langage défiguré et désinstrumentalisé de la poésie, Parents et amis se refusant donc à la calcification du texte et de ses mécanismes.

\section{Bibliographie}

ADRIAENSEN, Brigitte (2004), «L'ironie postmoderne et le retour de l'auteur », Texte, no 35-36, p. $79-104$.

AUDET, René (2013), « Une poétique illusionniste. Ironie de la forme et récit détourné (Éric Chevillard, Nicolas Langelier) », dans Didier Alexandre et Pierre Schoentjes (dir.), L'Ironie : formes et enjeux d'une écriture contemporaine, Paris, Classiques Garnier, coll. «Rencontres », p. 17-32.

BARTHES, Roland (1966), Critique et vérité, Paris, Éditions du Seuil.

BEAUdin-Gagne, David (2014), Procès-verbal; suivi de Filiation(s) rompue(s) : mémoire en pièces et tissus de parole dans Parents et amis sont invités à y assister d'Hervé Bouchard, mémoire de maîtrise, Montréal, Université de Montréal.

BOUCHARD, Hervé (2007), « Une affaire de retournement. Hervé Bouchard, causerie écrite II », entretien avec José Morel Cinq-Mars, Remue.net, http://remue.net/spip.php?article2323.

BOUCHARD, Hervé (2014 [2006]), Parents et amis sont invités à y assister. Drame en quatre tableaux avec six récits au centre, Montréal, Le Quartanier, coll. «Série QR ».

CAmus, Audrey (2012), « "Noir dans le théâtre où la scène est crayonnée”. La dramaturgie romanesque d'Hervé Bouchard », dans Nathalie Dupont et Éric Trudel (dir.), Pratiques et enjeux du détournement dans le discours littéraire des XXe et XXIe siècles, Québec, Presses de l'Université du Québec, p. 137-147.

CANTY, Daniel (2007), « Hervé Bouchard. Le livre de boue et la robe de bois », Liberté, vol. 49, no 3 , p. 63-78.

Deleuze, Gilles (1993), Critique et clinique, Paris, Éditions de Minuit, coll. « Paradoxe ».

ESTELLON, Vincent (2013), « Du complexe au syndrome de la "mère morte" », Les Cahiers du D.E.P.S. Revue en ligne du diplôme d'État de psychologie scolaire de l'Université Paris Descartes, https://lescahiersdudeps.wordpress.com/2013/05/13/du-complexe-ausyndrome-de-la-mere-morte-vincent-estellon/.

GoDIN, Louis-Daniel (2017), « Parler en décâliçant. Hervé Bouchard poursuit son exploration de la douleur de la mère », Liberté, no 315, p. 45-46. 
HutCheon, Linda (1986-1987), « The Politics of Postmodernism: Parody and History », Cultural Critique, no 5, p. 179-207.

INKEL, Stéphane (2008), Le paradoxe de l'écrivain. Entretien avec Hervé Bouchard, Saguenay, La Peuplade.

INKEL, Stéphane (2009), "Parler autour du Père mort. Les théâtres de paroles de Gérard Bessette et d'Hervé Bouchard», @nalyses, vol. 4, no 3, p. 119-140, https://uottawa.scholarsportal.info/ojs/index.php/revue-analyses/article/view/718/619.

KEMEID, Olivier (2007), «Lecteurs et amis sont invités à y assister », Liberté, vol. 49, n 3, p. 7988, http://id.erudit.org/iderudit/34653ac.

MAllarme, Stéphane (1897), « Crayonné au théâtre », dans Divagations, Paris, Eugène Fasquelle, p. 153-163.

Michaud, Ginette (1985), «Récits postmodernes? », Études littéraires, vol. 21, no 3, p. 67-88, https://www.erudit.org/fr/revues/etudfr/1985-v21-n3-etudfr1632/036870ar.pdf

OUELLET, François (2010), "Filiation et discours religieux dans le roman québécois contemporain. À propos de Parents et amis sont invités à y assister d'Hervé Bouchard", dans Zuzana Malinovská (dir.), Cartographie du roman québécois contemporain, Prešov (Slovaquie), Université de Prešov, coll. « Monographia », p. 201-217.

OuELLET TREMBLAY, Laurance (2016), «Ça ne finira pas, c'est parti pour l'éternité, fallait que vous le sachiez » : l'engendrement par la parole chez Hervé Bouchard, Pierre Perrault et Hector de Saint-Denys Garneau, thèse de doctorat, Montréal, Université du Québec à Montréal.

SCHOENTJES, Pierre (1993), Recherche de l'ironie et ironie de la recherche, Gand, Rijksuniversiteit te Gent, Universa Press.

Schoentjes, Pierre (2001), Poétique de l'ironie, Paris, Éditions du Seuil.

VIART, Dominique (1999), «Filiations littéraires », dans Jan Baetens et Dominique Viart (dir.), Écritures contemporaines 2 . États du roman contemporain, Paris, Lettres modernes Minard, p. $115-139$.

WILDE, Alan (1981), Horizons of Assent: Modernism, Postmodernism, and the Ironic Imagination, Baltimore, The Johns Hopkins University Press.

\section{Résumé}

Parents et amis sont invités à y assister d'Hervé Bouchard brouille les limites entre les genres littéraires, nous confronte aux discours religieux et familial désarticulés que le $\mathrm{xx}$ siècle a déjà mis en pièces et charcute la langue normalisée. Cette contestation des typologies génériques, des discours fédérateurs et de la langue standard se présente comme différents mécanismes 
d'une ironie postmoderne (Alan Wilde), c'est-à-dire une ironie qui ne vise plus à opposer à un discours supposément irrecevable un autre discours supérieur, mais bien à réfuter l'idée même de discours en mettant au jour la multiplicité de ce que l'on croyait stable.

\begin{abstract}
Parents et amis sont invités à y assister by Hervé Bouchard blurs the line between literary genres, confronts us with the disarticulated religious and familial discourses that the $20^{\text {th }}$ century has already torn to pieces and hacks the normative language. Challenging the traditional genre typologies, our federative social discourses, as well as standard French partakes of what Alan Wilde calls postmodern irony, that is, a type of irony that does not pit one supposedly unacceptable discourse against another, superior one, but rather refutes the concept of discourse itself by showing the multiplicity of what we believed to be stable.
\end{abstract}

